\title{
Capítulo 8 \\ La cuarta revolución y la era de la inteligencia artificial: implicaciones en la seguridad y el trabajo ${ }^{1}$ Carlos Enrique Álvarez Calderón*
Yesid Eduardo Ramírez Pedraza**
}

$1 \quad$ Este artículo hace parte del proyecto de investigación de la Maestría en Seguridad y Defensa Nacionales, titulado "Desafíos y nuevos escenarios de la seguridad multidimensional en el contexto nacional, regional y hemisférico en el decenio 2015-2025", el cual hace parte del grupo de investigación Centro de Gravedad de la Escuela Superior de Guerra General Rafael Reyes Prieto, reconocido y categorizado en (A1) por MINCIENCIAS, con el código COL0104976.

* Politólogo y magíster en Relaciones Internacionales de la Pontifica Universidad Javeriana. Coaching Ontológico Empresarial de la Universidad San Sebastián de Santiago de Chile. Coordinador de investigación en Seguridad y Defensa Nacionales de la Escuela Superior de Guerra de Colombia. Correo electrónico: carlos.alvarez@esdegue.edu.co

** Coronel en retiro del Ejército Nacional de Colombia, magíster en Seguridad y Defensa del Colegio Interamericano de Seguridad y Defensa. Director de la Maestría en Seguridad y Defensa Nacionales de la Escuela Superior de Guerra de Colombia. Correo electrónico: yesid.ramirez@esdegue.edu.co 


\section{CÓMO CITAR}

Álvarez Calderón, C. E., \& Ramírez Pedraza, Y. E. (2020). La cuarta revolución y la era de la inteligencia artificial: implicaciones en la seguridad y el trabajo. En Y. Rico, D. López, \& A. Cerón R. (comps.), Enfoques y gestión en Seguridad Integral (pp. 209-237). Escuela de Postgrados de la Fuerza Aérea Colombiana. https://doi.org/10.8667/9789585996199.08

\section{Colección Ciencia y Poder Aéreo N. 16}

ENFOQUES Y GESTIÓN EN SEGURIDAD INTEGRAL

\section{CAPÍTULO 8.}

La cuarta revolución y la era de la inteligencia artificial:

implicaciones en la seguridad y el trabajo

https://doi.org/10.8667/9789585996199.08

Bogotá, Colombia

Noviembre, 2020 


\section{RESUMEN}

Las tecnologías de la información, potencializadas por la cuarta revolución industrial y su amplia adopción en el mundo, vienen dando lugar a una serie de nuevos y revolucionarios modelos comerciales, así como al surgimiento de nuevos desafíos en términos de seguridad. Por ejemplo, en un ambiente económico y productivo cada vez más dependiente del ciberespacio y la automatización, la seguridad de la información debería considerarse como un componente crítico de la seguridad integral de las organizaciones, ya que su función debe ser establecer la confianza entre organizaciones e individuos y permitir que el intercambio de información a través de Internet sea seguro y proporcione a las personas la tranquilidad necesaria para realizar sus actividades productivas. Por lo tanto, este documento de reflexión busca dar cuenta de cómo los recientes cambios tecnológicos están transformando en el presente los aspectos sociales, políticos y económicos de la vida humana, pero, además, la gestión de la seguridad en todos sus niveles.

\section{PALABRAS CLAVE}

Industrialización; inteligencia artificial; revolución tecnológica; seguridad laboral; sociedad de la información. 


\section{Introducción}

Diversos estudios académicos (Jensen \& Wiest, 2001; Søgaard et al., 2009; Headrick, 2009; Teng, 2014; Álvarez et. al., 2017; Álvarez \& Zambrano, 2017), han dado cuenta de la importancia que el cambio tecnológico ha tenido a través de la historia en las creencias políticas y sociales, así como en la expansión del poder político y la riqueza material de las sociedades, un fenómeno que indudablemente antecede la reciente era digital.

Tradicionalmente, la tecnología se ha definido como la forma en que el conocimiento científico evoluciona en la producción de bienes, servicios y en el cumplimiento de objetivos, utilizando herramientas y técnicas para lograr ciertos resultados. El término "tecnología" se originó del uso que en el siglo Xvi se le dio a la palabra griega tekne

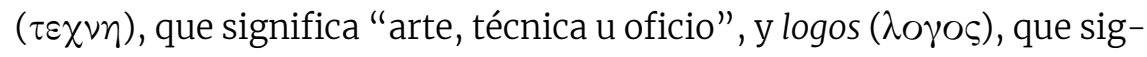
nifica "conjunto de saberes".

La historia de la relación entre la humanidad y la tecnología es extensa; se remonta millones de años atrás al primer uso humano de las piedras como herramientas y a su configuración en dispositivos más eficientes hacia el paleolítico temprano. Los pueblos primitivos descubrieron el uso del fuego como una tecnología de supervivencia e idearon sistemas cada vez más complejos de gestión del agua para el riego y posteriormente para la energía hidroeléctrica. Así mismo, a medida que las tecnologías de las comunicaciones se desarrollaban gracias a la difusión de la imprenta, el telégrafo o Internet, la gente pudo saber más sobre otros países, haciéndose más cosmopolita y conocedora de su entorno.

El periodo del renacimiento tardío en Europa occidental vio un gran progreso en las artes y las ciencias, así como la agitación social que unía a la Edad Media con la historia moderna. La tecnología cambió 
los conjuntos de habilidades humanas al implementar métodos y procesos para el manejo de los recursos naturales y de esta manera obtener ventajas en la competencia y adquisición de servicios. A su vez, la tecnología en las ciencias de la salud y la agricultura mejoró la esperanza de vida, en especial en la Edad Moderna; y aunque dichas tecnologías parecían tener una función exclusivamente personal, pues hacían la vida más placentera y eficiente, los líderes de antaño y de tiempos más recientes han recurrido a la tecnología como una ayuda para construir los Estados y conquistar a otros pueblos. En efecto, acueductos que se extienden por cientos de kilómetros y la construcción de barcos para la guerra y el comercio fueron algunas de las tecnologías que les permitieron a estos líderes mantener y expandir su poder en el sistema internacional. El armamento comparativamente simple de la gente en la Edad de Piedra dio paso a una maquinaria más compleja para la conquista y la destrucción, armamento que ha tenido un uso cada vez más devastador (Álvarez et. al., 2017).

Las limitaciones de la fuerza mecánica humana y los descubrimientos del fuego, metales e iluminación, propiciaron el desarrollo de herramientas que ayudarían o sustituirían el esfuerzo humano (Zafirovski, 2011). Por esta razón, las máquinas se construyeron con el fin de proporcionar un medio para que los humanos pudiesen operar y lograr ciertas tareas, o para reemplazar el esfuerzo humano en su totalidad. La maquinaria consumida y la energía convertida proporcionaron, por medios mecánicos, químicos, térmicos, eléctricos u otros, plusvalías económicas que transformaron a la sociedad, la economía $\mathrm{y}$, por supuesto, a la seguridad.

Dicho lo anterior, este documento de reflexión busca dar cuenta del impacto de los recientes cambios tecnológicos que van más allá de la computación tradicional y que el Foro Económico Mundial ha denominado la cuarta revolución industrial, la cual estaría transformando, 
en el presente, los aspectos sociales, políticos y económicos de la vida humana, pero, además, la gestión de la seguridad en todos sus niveles.

\section{Las primeras tres revoluciones}

La palabra "revolución" denota un cambio abrupto y radical. De acuerdo con Shwab (2016), las revoluciones se han producido a lo largo de la historia cuando nuevas tecnologías y formas novedosas de percibir el mundo desencadenan un cambio profundo en los sistemas económicos y las estructuras sociales. En este orden de ideas, el primer cambio estructural en la forma de vida de los seres humanos pudo haber llegado a presentarse con la sedentarización de las sociedades y la domesticación de animales, en el proceso de transición hacia la agricultura, hace unos 10.000 años. Antes de la mecanización, el esfuerzo humano fue impulsado por la existencia de animales y la mano de obra humana para construir, trabajar la tierra y viajar; en este contexto, la revolución agraria combinó el esfuerzo animal con el de los seres humanos para la producción, el transporte y las comunicaciones. Luego, con la acción mecánica del agua, el viento y el fuego, la productividad agrícola se incrementó exponencialmente, estimulando el crecimiento de la población y permitiendo asentamientos humanos más grandes, dando paso, hace aproximadamente 5.000 años, a la urbanización y al surgimiento de los primeros estados primitivos (Álvarez, 2017).

A partir de la segunda mitad del siglo xviII, la revolución agraria sería seguida por una serie de revoluciones industriales, las cuales "marcaron la transición de la potencia muscular a la potencia mecánica, evolucionando hacia la actual cuarta revolución industrial, en donde el poder cognitivo mejorado está aumentando la producción 
humana" (Schwab, 2016, p. 11). En términos generales, se aceptó que el término "revolución industrial" 2 se pudiese referir, en principio, al periodo comprendido entre 1770 y 1870, en el cual el cambio tecnológico permitió aprovechar las fuerzas mecánicas y eléctricas para las transformaciones de los métodos de fabricación y producción que, aunados a nuevos modos de transporte y modernos tipos de infraestructura, abandonaron la lógica feudal de una economía dependiente de la actividad agrícola. Si bien su génesis se produciría en Occidente, particularmente en Gran Bretaña (transformando el Imperio Británico en el "taller del mundo"), en un siglo ya se había extendido al continente americano y a la cuenca del Asia y el Pacífico.

La primera revolución industrial, desencadenada por la construcción de ferrocarriles y la invención de la máquina de vapor, marcaría el comienzo de la producción mecánica. La primera máquina de vapor fue construida por Tomas Savery en 1698, y se utilizó para bombear agua acumulada de las minas de carbón; a pesar de la maravilla tecnológica que representaba para la época, tenía una aplicación limitada, ya que utilizaba presión atmosférica y trabajaba en contra del vacío del vapor condensado para extraer agua. Sin embargo, el salto de la presión de vacío, pasando por la energía cinética mecánica, hasta el movimiento continuo de rotación, no se produciría sino hasta 1781 con el advenimiento del diseño revolucionario de James Watt para su máquina de vapor.

Su motor permitió alimentar una amplia gama de fabricación, maquinaria agrícola y de producción. El invento marcó el comienzo de lo

2 El término "revolución industrial" ingresó por primera vez en el léxico del pensamiento en 1799 (Cipolla, 2003).

3 Posteriormente, en 1712, Tomas Newcomen desarrollaría la primera máquina de vapor comercial basada en un diseño de pistón. 
que se describiría como la primera revolución industrial y el inicio de la mecanización al permitir la producción de energía mecánica a partir de la energía térmica generada por la combustión de productos químicos y el oxígeno. Su revolución fue la capacidad de aprovechar la energía mecánica sin el uso de intervención humana o animal y facilitar a los seres humanos trabajar más eficazmente usando energía mecánica (desde bombas estacionarias fijas, elevadores de grúas y molinos, hasta la locomoción en forma de trenes y carruajes sin caballos). En consecuencia, y hacia 1886, las máquinas de vapor ya serían capaces de desarrollar 10.000 caballos de fuerza, utilizándose a gran escala en barcos de vapor interoceánicos y locomotoras industriales de largo alcance (Jörg, 2016).

La segunda revolución industrial, de finales del siglo xIX y principios del siglo xx, hizo posible la producción en masa, fomentada por el advenimiento de la cadena de montaje, los motores eléctricos y la electrificación a escala industrial. Adicionalmente, el motor de combustión petroquímico y el prototipo inicial del moderno motor de gasolina habilitaron la construcción del primer automóvil en 1885 por parte de Gottlieb Daimler.

La tercera revolución industrial se daría a partir de 1960. Suele denominarse revolución "informática" o "digital", ya que se caracterizó por el desarrollo de los semiconductores (década de 1960), la informática personal (años 70 y 80), e Internet (década de 1990). Sus inicios se establecieron con los desarrollos en la microelectrónica y los semiconductores de mediados de la década de 1950 hasta principios de los años setenta, cuando los primeros procesos de integración a gran escala crearon circuitos integrados, combinando miles de transistores en un solo chip (Cooper \& Kaplinsky, 1989).

El circuito integrado aceleró el paso desde la tecnología mecánica y analógica a la electrónica digital, transformando la digitalización 
de la información y los procesos informáticos, que, como resultado, alentaron el surgimiento de la informática empresarial, liderada por compañías como IBM, Hewlett Packard, Microsoft, Sun Microsystems y una plétora de diferentes empresas. Eventualmente, el desarrollo de las telecomunicaciones y su infraestructura condujeron al inicio de Internet en la década de 1990, que hacia finales de esta ya había creado los cimientos de los centros de datos mundiales, los dispositivos móviles y la aparición de los motores de búsqueda (como Google), los mercados en línea (como Amazon o Apple Store), las redes sociales (como Facebook y Twitter), entre muchas otras plataformas, extendiendo la revolución digital a todos los rincones del mundo y conectando a personas e industrias en una escala sin precedentes.

\section{La cuarta revolución industrial: implicaciones en el trabajo}

El nacimiento de la World Wide Web trajo consigo una nueva sintaxis y protocolos que permitieron a las maquinas "hablar" entre ellas y con los humanos; a su vez, los acelerados avances en la inversión de espectro y ancho de banda proporcionaron enlaces a empresas comerciales y ciudades, a las infraestructuras de redes de transporte, energía y servicios públicos. No obstante, desde comienzos del siglo xxI, nuevos avances tecnológicos en inteligencia artificial, impresión 3D, robótica, computación cuántica, nanotecnología y bioingeniería, entre otras, estarían cambiando radicalmente la manera de fabricar, intercambiar y consumir materiales, dinero, productos y servicios.

Este conjunto de tecnologías dio cuenta de una cuarta revolución industrial. Si bien parten de la revolución digital, es la fusión de estas tecnologías y su interacción a través de los dominios físicos, digitales 
y biológicos lo que hace que la cuarta revolución industrial sea fundamentalmente diferente de las revoluciones anteriores (Shwab, 2016).

En esta cuarta revolución, las tecnologías emergentes y la innovación de amplia base se difunden mucho más rápido y más ampliamente que en las anteriores, que continúan desarrollándose en algunas partes del mundo. La segunda revolución industrial aún no se ha experimentado por completo en el $17 \%$ del mundo, ya que casi 1.300 millones de personas aún carecen de acceso a la electricidad. Esto también es cierto para la tercera revolución industrial, con más de la mitad de la población mundial (4.000 millones de personas), la mayoría de las cuales vive en el mundo en desarrollo, sin acceso a Internet. Sin embargo, mientras el huso (el sello distintivo de la primera revolución industrial), tardó casi 120 años en extenderse fuera de Europa, por el contrario, el Internet permeó en todo el mundo en menos de una década (Shwab, 2016, pp.12-13).

Los avances técnicos en las ciencias de materiales, nuevas técnicas de fabricación, inteligencia artificial, investigación biológica, el internet de las cosas, las ciudades inteligentes, los automóviles sin conductor, la telemedicina, entre otros, han permitido desarrollos dentro de la cuarta revolución que tendrían el potencial de cambiar industrias enteras y la experiencia humana. Pero la cuarta revolución industrial también destaca la paradoja de estas tecnologías disruptivas y las nuevas habilidades que cambiarían la productividad a través de la automatización, planteando uno de los mayores desafíos al empleo humano.

Durante siglos, la llegada de nuevas tecnologías al lugar de trabajo ha provocado temores entre los trabajadores $\mathrm{y}$, a veces, reacciones violentas. Ya en 1589 la reina Isabel I de Inglaterra se negó a otorgarle una patente a un bastidor inventado por William Lee porque supuestamente estaba preocupada por el efecto que este tendría en el gremio de los tejedores a mano. A principios del siglo XIX, los trabajadores 
textiles de Gran Bretaña y Francia rompieron telares automáticos en sus fábricas y los impresores manuales protestaron la llegada de las prensas a vapor.

Desde la Primera Revolución Industrial en el siglo XviII, las economías de Europa, Estados Unidos y otros países sufrieron dos oleadas de cambios estructurales. Durante la primera oleada, la mecanización permitió una revolución en la agricultura y en la industria, lo que provocaría una migración de los trabajadores del campo a las ciudades; la segunda se produjo en los últimos 60 años, ya que la participación del empleo en el sector manufacturero ha disminuido en algunos países, incluso cuando el crecimiento en los sectores de servicios se aceleró (Herrendorf et al., 2014). Los cambios de los empleos que han venido acompañando este proceso de transformación estructural han sido relevantes; por ejemplo, en los Estados Unidos la participación de la agricultura en el empleo disminuiría del $58 \%$ del empleo total en 1850, al 2,5\% del empleo en la actualidad. En solo 40 años, entre 1880 y 1920, la participación del empleo agrícola disminuyó un 25 \%; durante las mismas décadas, otros sectores también se transformaron por la mecanización y la electrificación, como, por ejemplo, la disminución en la proporción de mineros y trabajadores domésticos.

Desde 1960, cuando comenzó la segunda ola de transformación estructural, la fabricación cayó del 27 \% al 9 \% del empleo total en los Estados Unidos, a medida que la automatización y el comercio mundial transformaron la fabricación y la demanda de servicios. En general, los patrones son similares en otros países; el empleo agrícola en China cayó como porcentaje del empleo total el $32 \%$ en solo 25 años, del $60 \%$ en 1990, al $28 \%$ en el 2015. En México, la participación de la agricultura en el empleo disminuyó del 52 \% en 1960 al $13 \%$ en el 2015. En Japón, el empleo agrícola disminuyó de una proporción del $31 \%$ del empleo total en 1960 al 3,5\% en el 2015 (Manyika et al., 2017). 
Desde David Ricardo hasta Karl Marx y John Maynard Keynes, expresaron en su momento las preocupaciones por el efecto del cambio tecnológico en el empleo, como sucede en el presente con los rápidos avances en robótica e inteligencia artificial. El economista político David Ricardo estaba preocupado a principios del siglo XIX porque las máquinas harían que el trabajo fuera redundante (Ricardo, 2004), mientras que Karl Marx preveía, en 1850, una era en la que los medios de trabajo serían transformados por "un sistema automático de maquinaria" (Marx, 1973). En 1930, John Maynard Keynes acuñó el término "desempleo tecnológico" para describir una situación en la que la innovación que economizaba en el uso del trabajo desarticulaba el ritmo al que podían crearse nuevos empleos, en una "fase temporal de inadaptación" (Keynes, 1963). En consonancia, varios académicos y tecnólogos destacados como Brynjolfsson y McAfee (2014) sostienen que la última ola de tecnologías podría ser particularmente perturbadora para el mercado laboral a nivel mundial en los próximos años.

\section{Las cuatro revoluciones industriales}

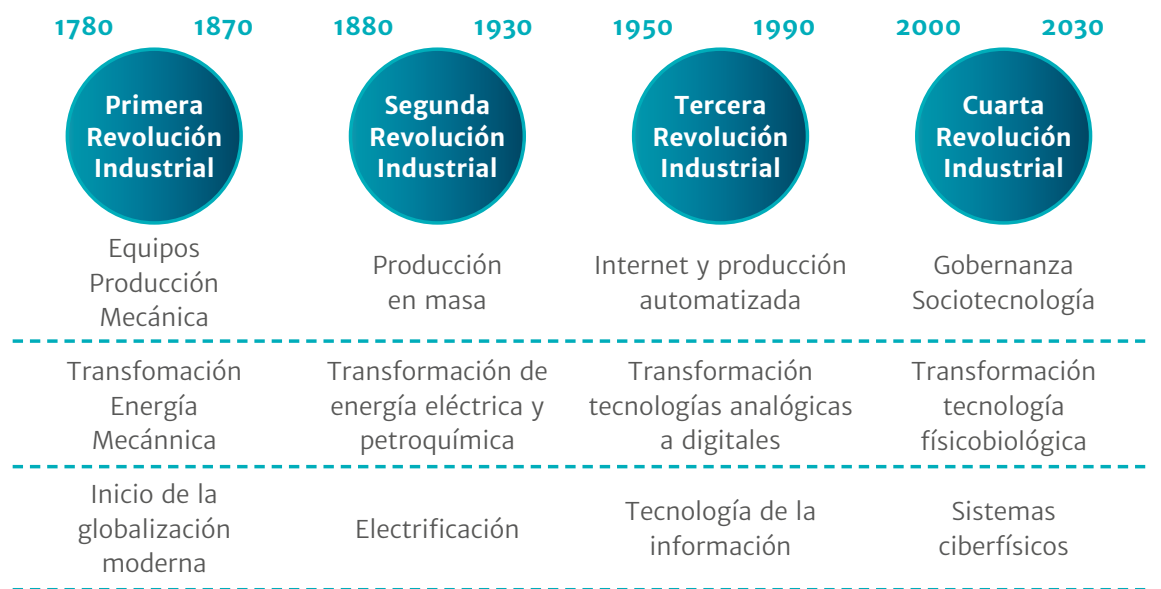

Figura 1. Evolución de las Revoluciones Industriales

Fuente: Adaptado de Skilton \& Hovsepian (2018). 
Hoy por hoy, algoritmos dictan la negociación automatizada de billones de dólares en activos de los mercados financieros, mientras los chatbots artificialmente inteligentes estarían desplazando a los humanos en los centros de atención telefónica o call center. Muy pronto los aviones y los automóviles podrían funcionar de manera autónoma, poniendo en peligro los medios de subsistencia de aquellos que conducen profesionalmente. A pesar de que los robots han venido realizando ciertos trabajos repetitivos en diversas fábricas durante décadas (como es el caso de las plantas ensambladoras de automóviles), ahora podrán "voltear" la carne de hamburguesas en la parrilla, descartar tomates verdes en una máquina clasificadora de alta velocidad usando reconocimiento de imágenes, o incluso colocar ladrillos en obras de construcción; inclusive, impresoras gigantes 3D en la actualidad podrían hacer casas de hormigón en una fracción del tiempo de lo que le tomaría a un equipo de obreros experimentados.

La Federación Internacional de Robótica afirmaba que en los procesos de fabricación, en el 2018, existía un promedio de 99 unidades de robots industriales por cada 10.000 empleados, en comparación con 74 unidades en el 2016. En el 2018, la instalación global de robots industriales aumentó un $6 \%$ con relación al 2017, alcanzando las 422.271 unidades y una cifra de US\$16,5 billones; el principal motor del crecimiento sería, al igual que en el pasado, la industria eléctrica y de electrónica (un incremento del $41 \%$ ). No obstante, la industria automotriz sigue constituyéndose como el principal cliente de robots industriales, participa del $30 \%$ del total de instalaciones, seguido por la industria eléctrica y electrónica con el $25 \%$, la industria metalmecánica con el $10 \%$, la industria de plásticos y químicos con el $5 \%$, y la industria de comidas y bebidas con el $3 \%$ (el $19 \%$ restante se distribuye en una diversidad de sectores industriales y agroindustriales) (Wittmann, 2019). 
Los 5 principales mercados de robots industriales están en China, Japón, Estados Unidos, Corea del Sur y Alemania, y concentran el 74 \% de las instalaciones globales de robots industriales. Según Wittmann (2019), China es el principal mercado desde el 2013, representando el $36 \%$ del total de las instalaciones en el 2017 y el 2018; tan solo en el 2018, se instalaron en China 154.032 unidades de robots industriales, una cantidad que excede el número combinado de robots industriales instalados en el continente americano y europeo (130.772 unidades). Los siguientes mercados de importancia en la instalación de robots industriales son Japón, con 55.240 unidades y los Estados Unidos, con 40.373 unidades instaladas en el 2018.

Por su parte, el número total de robots de servicios profesionales vendidos en el 2018 aumentó en un $61 \%$, a 271.000 unidades, de los 168.000 en el 2017, alcanzando la cifra de US\$9,2 billones. Los vehículos autónomos, particularmente los vehículos aéreos no tripulados, representan la fracción más grande en el mercado de robots de servicio profesional ( $41 \%$ de todas las unidades vendidas). La segunda categoría más grande son los robots de inspección y mantenimiento, participan del $39 \%$ de todas las unidades vendidas. Por su parte, los robots de servicio para aplicaciones de defensa representaron el $5 \%$ del número total de robots de servicio vendidos en el 2018 (Haegele, 2019). Con relación a las ventas de exoesqueletos humanos motorizados, estas aumentaron de 6.700 unidades en el 2017 a 7.300 unidades en el 2018 y su creciente demanda se debe a que los exoesqueletos respaldan el trabajo ergonómico al reducir las cargas en el trabajador.

A pesar de que se encuentran entre los robots de servicio más caros del mercado, se ha presentado una importante dinámica en las ventas de robots médicos, pasando de 3.400 unidades en el 2017 a 5.100 unidades vendidas en el 2018 (un aumento del 50 \%). Las aplicaciones 
médicas más importantes de robots de servicios son la cirugía asistida por robot o los sistemas de terapia y rehabilitación (Haegele, 2019). Los robots también han jugado un papel importante en la lucha contra el covid-19 en todo el mundo; por ejemplo, el robot de desinfección UVD ha tenido una gran demanda desde el brote de la pandemia de CoviD-19 en el 2020, hasta el punto de que se ordenaron más de 2.000 robots UVD para destruir el virus en los hospitales de China, y particularmente en la ciudad de Wuhan, el epicentro de la pandemia. Con relación a lo anterior, los robots de servicio para uso personal y doméstico se encuentran principalmente en las áreas de robots domésticos (aspiración, limpieza de pisos, corte de césped, limpieza de piscinas) y robots de entretenimiento (juguetes, sistemas de pasatiempos, educación e investigación). El número total de robots de servicio para uso personal y doméstico aumentó en un 59 \% en el 2018 con relación al 2017; tan solo en el 2018, se vendieron más de 11,6 millones de robots aspiradores y limpiadores de pisos (Haegele, 2019).

En suma, la automatización del software informada por el aprendizaje automático y la inteligencia artificial (IA) tendría un profundo efecto en el mercado laboral. Los chatbots inteligentes parecerían reemplazar a la mayoría del personal de los call center en unos 10 años, hasta el punto de que, para el 2020, el $85 \%$ de las preguntas podrían llegar a ser respondidas por asistentes virtuales; cuando se tiene en cuenta que una gran compañía de telecomunicaciones como AT\&T emplea alrededor de 100.000 operadores de call center para atender a sus 120 millones de clientes, se evidencia que serían muchos los trabajos que podrían desaparecer rápidamente como consecuencia de la cuarta revolución industrial. En Colombia, el impacto también sería notorio, tomando en cuenta que esta industria que factura $\$ 2,9$ billones de pesos, empleaba en el 2014 unas 180.000 personas. 
Manyika et al. (2017) concluyen que casi dos tercios de todos los puestos de trabajo podrían tener una parte significativa de sus actividades automatizadas para el 2030 (al menos un 30 \%), afectando 800 millones de puestos de trabajo. La automatización podría acelerar la productividad de la economía mundial entre 0,8 y 1,4 \% del Producto Interno Bruto (PIB) global al año, suponiendo que la mano de obra humana reemplazada por la automatización vuelva a unirse a la fuerza de trabajo y sea tan productiva como lo fue en el 2014. La automatización por sí sola no sería suficiente para lograr aspiraciones de crecimiento económico a largo plazo en todo el mundo; para eso, se necesitarían medidas adicionales que aumenten la productividad, incluida la reelaboración de procesos comerciales o el desarrollo de nuevos productos y servicios. No obstante, el crecimiento de la productividad que permite la automatización puede garantizar la prosperidad continua en las naciones envejecidas y proporcionar un impulso adicional a las de rápido crecimiento. Para las empresas, la implementación de la automatización podría brindar beneficios en el ahorro de costos laborales, pero también en una miríada de otras formas de mejorar el rendimiento. Puede facilitarles a las empresas acercarse a los clientes y predecir las necesidades de mantenimiento, reduciendo drásticamente el costo de las operaciones en algunas actividades y ampliando la vida útil de los activos de capital existentes.

\section{La inteligencia artificial: implicaciones en la seguridad}

La inteligencia artificial (IA) podría ser definida como "cualquier sistema artificial que realice tareas en circunstancias variables e impredecibles, sin supervisión humana significativa, o que pueda aprender 
de su experiencia y mejorar su rendimiento [...], pudiendo resolver tareas que requieren percepción, cognición, planificación, aprendizaje, comunicación, o acción física" (Ayoub \& Payne, 2016, p. 812).

Si bien el campo de la investigación en IA comenzó en $1956^{[4]}$, su interés se vería potencializado en el 2010 gracias a la convergencia de tres desarrollos habilitantes: la disponibilidad de fuentes de Big Data5, mejoras en los enfoques de aprendizaje automático y el aumento en el poder de procesamiento de la computadora. Este crecimiento ha avanzado el estado de la IA "modular", que se refiere a algoritmos que abordan conjuntos de problemas específicos, como juegos, reconocimiento de imágenes y vehículos autónomos (todos los sistemas actuales de IA caen dentro de la categoría de la IA modular). El enfoque más prevalente para la IA modular es el aprendizaje automático, que implica algoritmos estadísticos que replican las tareas cognitivas humanas derivando sus propios procedimientos a través del análisis de grandes conjuntos de datos de entrenamiento. Durante el proceso de capacitación, el sistema informático crea su propio modelo estadístico para realizar la tarea especificada en situaciones que no ha encontrado anteriormente.

Las IA modulares tienen una experiencia limitada en un dominio particular y pueden aprender a través de la práctica para mejorar su rendimiento. Una IA general, por el contrario, puede utilizar su

4 En 1956, John McCarthy propuso organizar un taller de dos meses sobre la idea de la recién creada inteligencia artificial. La reunión estimuló la investigación que condujo primero a sistemas automáticos y luego a sistemas expertos en los años 70 y 80 , respectivamente. El primero implicaba la automatización simple de máquinas de preguntas matemáticas y estadísticas, incluida la resolución de pruebas lógicas.

5 Los Big Data o Metadatos son aquellos conjuntos de datos o combinaciones de conjuntos de datos cuyo tamaño (volumen), complejidad (variabilidad) y velocidad de crecimiento (velocidad) dificultan su captura, gestión, procesamiento o análisis mediante tecnologías y herramientas convencionales, tales como bases de datos relacionales y estadísticas convencionales. La mayoría de los analistas y profesionales actualmente se refieren a los Big Data como los conjuntos de datos que van desde 30-50 Terabytes a varios Petabytes. 
conocimiento de forma mucho más flexible para abordar una gama mucho más amplia de problemas más abstractos y sin límites, incluidos los que requieren una comprensión de los significados y valores. Tanto la IA modular como la general son capaces de aprender acerca de la tarea en la que participan y de mejorar su desempeño a lo largo del tiempo. Una IA modular que trabaja para mejorar el rendimiento de su dominio es similar a un golfista que perfecciona su swing, o a un guitarrista virtuoso que trabaja en su solo; la máquina aprende y se adapta, pero su arquitectura subyacente (hardware y software por igual) permanece sin cambios.

Los expertos generalmente coinciden en que pasarán muchas décadas antes de que se pueda avanzar a un estado de IA general, o a sistemas capaces de inteligencia a nivel humano en una amplia gama de tareas. Sin embargo, el creciente poder de los algoritmos de IA modulares ha provocado una ola de interés comercial. La inversión en IA está creciendo rápidamente, dominada por gigantes digitales como Google y Baidu; por su parte, Manyika et al. (2017) estimaron que a nivel mundial los gigantes tecnológicos gastaron entre US\$20 mil millones a US\$30 mil millones en IA en el 2016, con un $90 \%$ de este gasto en Investigación y Desarrollo (I+D) y un $10 \%$ en adquisiciones de IA.

En el marco de las guerras de quinta generación (Álvarez et. al., 2017), la IA tendría una serie de características únicas que podría ser importante a considerar a medida que estas tecnologías ingresaran al campo de la seguridad nacional. Primero, la IA es una tecnología de uso múltiple, ya que tiene el potencial de integrarse en prácticamente todo lo que se estime; Kelley (2014) declaraba que la IA "animará a los objetos inertes, como lo hizo la electricidad hace más de un siglo. Todo lo que antes electrificamos ahora lo cognitizaremos" (p. 23). En segundo lugar, muchas aplicaciones de iA serían de doble uso, es decir, que tienen aplicaciones militares y civiles. Por ejemplo, 
"los algoritmos de reconocimiento de imágenes pueden entrenarse para reconocer gatos en videos de YouTube y actividades terroristas en videos en movimiento (FMV) capturados por aviones piloteados remotamente (RPA) en Siria o Afganistán" (Allen \& Chan, 2017, p. 47). En tercer lugar, la IA es relativamente transparente, lo que significa que su integración en un producto no sería inmediatamente reconocible.

A nivel táctico, las ventajas de la IA modular le otorgarían ventajas a un actor de ganar las batallas donde pueda desplegarse efectivamente. Una IA modular que puede optimizar alguna actividad táctica (por ejemplo, el asalto a una posición enemiga, la coordinación rápida de ataques y la maniobra a través de plataformas automatizadas en red), superaría con creces a un comandante de batallón experimentado. El poder marítimo y aéreo serían los dominios más susceptibles a la IA debido a la falta comparativa de la compleja intervención humana relativa, por ejemplo, a la guerra urbana irregular (Álvarez, 2017). No obstante, existe un riesgo a que la IA no esté libre de errores; en 1988, el sistema de puntería de un destructor estadounidense equipado con Aegis derribó por error a un avión civil iraní, identificándolo como un caza F-14 (Singer, 2004). En este sentido, una capacidad táctica tuvo un impacto estratégico. En el futuro, las decisiones similares sobre la identidad de los posibles adversarios serán cada vez más automatizadas, sobre todo porque los sistemas opuestos de otra manera serán tecnológicamente capaces de frustrar a los operadores humanos. Un ataque de enjambres de ágiles y autónomos actores solo podría defenderse con sistemas que operen rápidamente, con autonomía e inteligencia.

En el nivel de toma de decisiones estratégicas, la IA modular podría mejorar la calidad de la toma de decisiones humanas a niveles estratégicos, utilizando sus capacidades para modelar a través de micromundos, evaluando riesgos. La máquina podría dominar grandes 
cantidades de datos, reconocer patrones que de otro modo podrían pasarse por alto y desafiar las suposiciones aceptadas. Podría responder a situaciones dinámicas más rápidamente y con menos deficiencias cognitivas que un estratega humano. La IA podrá evitar algunos de los defectos humanos de la estrategia, como la susceptibilidad a invertir en costos irrecuperables o el razonamiento afectivo, incluido el juicio de riesgo sesgado. Puede mitigar los efectos de la fatiga y el estrés, así como la carga de sobrecarga de información (Ayoub \& Payne, 2016). Una IA modular que actúa como consejero estratégico puede conferir una ventaja distintiva anticipando e identificando riesgos con opciones estratégicas, incluidas aquellas que los humanos no han previsto; y podría aprender de sus errores y mejorar sus algoritmos a medida que avanza el conflicto, mientras que los humanos permanecen enraizados en su heurística cognitiva y arraigado pensamiento grupal.

Un actor protagónico en el conflicto ideológico que caracterizarían las guerras de redes (Net War), en el marco de guerras de quinta generación, serían las redes sociales (Álvarez et. al., 2017). Las redes sociales se describen como el software que permite a las personas interactuar, conectarse, jugar o colaborar mediante el uso de una red informática. Esta definición abarca los sitios populares de redes sociales, incluidos blogs, wikis, podcasts, etiquetas y, más recientemente, los motores de búsqueda. Incluso hace relativamente poco, el uso de comunidades en línea para establecer y construir conexiones entre aquellos con intereses compartidos también se ha convertido en parte del mundo corporativo. A medida que las redes sociales profesionales como LinkedIn y Blue Chip Expert continúan creciendo, y los grupos profesionales ganan popularidad en sitios que alguna vez fueron personales como Facebook y MySpace, los profesionales de la seguridad integral y la gestión de riesgos deberán enfrentar la realidad de que 
estos sitios están surgiendo como fuente de divulgación no autorizada de información corporativa confidencial.

Si bien existen numerosos beneficios para las soluciones de redes sociales, el profesional en seguridad integral debería centrarse en los riesgos de las redes sociales. Al igual que con todas las tecnologías emergentes, las redes sociales avanzan rápidamente y los profesionales de la seguridad deben ser conscientes de los riesgos asociados. Hay una generación que ingresa a la fuerza de trabajo que supone que esta tecnología no solo estará disponible para su uso, sino que también sería esencial para la forma en que se comunican con colegas y socios comerciales. Aunque la utilización de las redes sociales conlleva ventajas internas y externas, la política y la arquitectura para defenderse de los riesgos deben abordarse de manera proactiva y no tomarse a la ligera.

En las transacciones comerciales, la integridad y la confidencialidad son ingredientes clave en la construcción de la confianza mutua. Del mismo modo, para realizar negocios a través de Internet, las empresas deben tener la seguridad de que cualquier información transmitida a través de Internet no será manipulada o robada. Dichos incidentes tendrían un efecto devastador en la credibilidad de todas las transacciones comerciales futuras y, en última instancia, podrían socavar las relaciones comerciales. Por ello, es críticamente importante que las empresas estén equipadas con mecanismos que aseguren la integridad de su información y comunicaciones digitales; este es un requisito absolutamente vital para la promoción y preservación de la confianza dentro de una empresa, así como también entre la compañía, sus clientes y socios comerciales.

Como resultado, el advenimiento de Internet fue rápidamente seguido por un gran interés y preocupación con respecto a la seguridad de la información. El éxito de la seguridad se basaría en la combinación 
correcta de personas, procesos, políticas y tecnología de un sistema de gestión de red con la capacidad de razonamiento intelectual, toma de decisiones dinámicas en tiempo real y auto-adaptación y mejoras basadas en la experiencia. Por ende, el diseño de un marco de gestión de redes sociales eficiente, dinámico y automatizado requeriría el apoyo del campo de la IA. Lidiar con la incertidumbre y la inconsistencia ha sido parte de la IA desde sus orígenes; en efecto, las tecnologías para gestionar la incertidumbre y la inconsistencia ya se han utilizado en áreas como los algoritmos de clasificación utilizados en los motores de búsqueda web. La expectativa es que las tecnologías de iA puedan desempeñar un papel igualmente importante en el contexto de las evaluaciones de seguridad.

El campo de la IA involucra el diseño e implementación de sistemas que exhiben capacidades de la mente humana, tales como el razonamiento, el conocimiento, la percepción, la planificación, el aprendizaje y la comunicación. La IA abarca varias subdisciplinas que incluyen aprendizaje automático, satisfacción de restricciones, sistemas de búsqueda y agentes múltiples, razonamiento e ingeniería y procesamiento del lenguaje natural. Las técnicas basadas en los principios de IA como red neuronal, algoritmo genético, sistemas expertos e inferencia difusa, proporcionan habilidades sofisticadas de toma de decisiones inteligente, mejora basada en la experiencia y resolución creativa de problemas. Por consiguiente, su aplicación en aspectos de las métricas de seguridad podría ser beneficiosa, particularmente como un medio para reducir la subjetividad y la participación humana en la realización de evaluaciones de seguridad (Sattikar \& Kulkarni, 2012).

La técnica de inteligencia artificial más nueva, como los Procesadores de Lenguaje Natural (PLN), se parece mucho a la forma en que aprenden los cerebros humanos. Además de las operaciones comunes 
del procesador de textos que tratan el texto como una mera secuencia de símbolos, los PLN consideran la estructura jerárquica del lenguaje: varias palabras forman una frase, varias frases hacen una oración y, en última instancia, las oraciones transmiten ideas. Al analizar el significado de la lengua, los sistemas de PLN llevan mucho tiempo desempeñando funciones útiles, como corregir la gramática, convertir la voz en texto y traducir automáticamente entre idiomas. Del mismo modo, los PLN que en realidad son una matriz de algoritmos complejos, se pueden usar para detectar correos no deseados, para escanear mensajes de correo electrónico, descubrir el contenido de los mensajes en el cual el correo electrónico sospechoso se envía a un área de cuarentena donde un administrador puede ver el contenido para determinar si lo descarta o lo pasa (Sattikar \& Kulkarni, 2012).

Del mismo modo, las técnicas de IA también pueden ayudar a identificar el comportamiento intrusivo, utilizando técnicas de detección de anomalías y de detección de uso indebido. Las redes sociales son excelentes plataformas para aplicar las técnicas de IA. A medida que las redes sociales se hacen cada vez más grandes y las personas las utilizan para compartir más información, las técnicas de IA podrían ser realmente útiles para organizar la información y llevar las piezas más relevantes a los usuarios de una manera completamente personalizada, ayudando además a delinear las categorías básicas de las inquietudes en torno a la privacidad de la información, así como a su protección.

\section{Conclusiones}

La historia no necesariamente se repite, pero sí proporciona un valioso contexto y lecciones aprendidas para el futuro de la demanda laboral en un momento de automatización. Entre esas lecciones, la innovación 
tecnológica en el pasado ha permitido la creación de nuevos empleos, más de los que ha destruido, aumentando la productividad, estimulando los aumentos sostenidos en los niveles de vida y provocando un cambio en el equilibrio entre el trabajo y el ocio. Sin embargo, la transición no siempre ha sido fluida, por ejemplo, los salarios reales se estancaron durante casi 50 años en la Inglaterra del siglo XIX durante la Revolución Industrial, y solo volvieron a aumentar en un momento de reformas sustanciales de la política social.

Las nuevas tecnologías han aumentado el crecimiento de la productividad, lo que ha permitido a las empresas bajar los precios para los consumidores, pagar salarios más altos o distribuir beneficios entre los accionistas. Esto estimula la demanda en toda la economía, impulsando la creación del empleo. Sin embargo, la revolución industrial de finales del siglo Xvir vio la mecanización arrasar a muchas industrias. La agricultura en particular, que representaba alrededor del $50 \%$ de los puestos de trabajo en toda Europa, observó ese porcentaje disminuir a menos del $5 \%$ en la actualidad. Tal trastorno fue indudablemente doloroso para aquellos que no pudieron adaptarse a los nuevos avances, aunque eventualmente surgieron nuevos tipos de empleo.

Más recientemente, hubo cambios sísmicos en la economía global; en los últimos 30 años se ha venido experimentando una transformación digital con el surgimiento de Internet y los avances significativos en la globalización (Álvarez \& Zambrano, 2017). Los datos del Banco Mundial muestran que el desempleo global como porcentaje del total de la fuerza de trabajo cayó del 6,1 \% en 1991 al 5,8 \% en el 2017, a pesar de que la población aumentó de 5,4 mil millones a 7,6 mil millones en el mismo periodo. Por lo tanto, las tecnologías de la cuarta revolución industrial, incluidas la inteligencia artificial y la robótica, 
generaron beneficios significativos para los usuarios, las empresas y las economías, elevaron la productividad y el crecimiento económico de los Estados.

La Automatización Robótica de Procesos (ARP) eliminaría la necesidad de que el personal realice actividades aburridas, repetitivas y basadas en reglas, como ingresar datos o manejar la nómina, por lo que la ARP no tendría que conducir a un sacrificio del personal, ya que podría empoderarlos y darle rienda suelta a su creatividad, librándolos de hacer cosas improductivas. En este orden de ideas, las reuniones versarían sobre las ideas, y no tanto sobre mecánica o procesos. Pero incluso los optimistas admiten que a medida que desaparecen los trabajos poco calificados, la gente tendría que aprender nuevas habilidades para compensar. No obstante, el grado en que estas tecnologías desplacen a los trabajadores dependería del ritmo de su desarrollo y adopción, así como del crecimiento económico y del crecimiento de la demanda laboral. Aun cuando la cuarta revolución industrial afectaría algunas ocupaciones, también crearía nuevas ocupaciones que no existen hoy en día, al igual que lo hicieron las tecnologías del pasado. Sin embargo, para el 2030, entre 75 a 375 millones de trabajadores (del $3 \%$ al $14 \%$ de la fuerza laboral mundial) necesitarían cambiar de categoría ocupacional.

Además, todos los trabajadores deberán adaptarse a medida que sus ocupaciones evolucionen junto con máquinas cada vez más capaces; parte de esa adaptación requeriría un mayor logro educativo, o pasar más tiempo en actividades que requieren habilidades sociales y emocionales, creatividad, capacidades cognitivas de alto nivel y otras habilidades relativamente difíciles de automatizar. En consecuencia, para lograr buenos resultados durante la cuarta revolución industrial, los responsables de las políticas públicas y los líderes empresariales 
deberán abrazar los beneficios de la automatización y, al mismo tiempo, abordar las transiciones de los trabajadores generadas por estas tecnologías.

Estos cambios desafiarán los modelos actuales de capacitación de la fuerza de trabajo, así como los enfoques comerciales para el desarrollo de habilidades. Otra prioridad sería repensar y fortalecer la transición y el apoyo a los ingresos para los trabajadores atrapados en las corrientes opuestas de la automatización. En total, se exigiría la necesidad de un nuevo tipo de pensamiento y liderazgo que reconozca el desafío de gestionar los nuevos tipos de automatización y su seguridad, así como reconciliar las paradojas y otros fenómenos que han surgido como consecuencia de la cuarta revolución industrial.

Las tecnologías de la información, potencializadas por la cuarta revolución industrial y su amplia adopción en todo el mundo, vienen dando lugar a una serie de nuevos y revolucionarios modelos comerciales para la era moderna. De hecho, las tecnologías de la información están transformando las formas tradicionales de llevar a cabo negocios, reemplazándolos con soluciones que promueven el intercambio eficiente de información entre las partes involucradas y aseguran la satisfacción del cliente. En este orden de ideas, la seguridad de la información debería considerarse como un componente crítico de la seguridad integral de las organizaciones, ya que su función sería establecer la confianza entre organizaciones e individuos, permitiendo que el intercambio de información a través de Internet sea seguro y proporcione a las personas la tranquilidad necesaria para realizar negocios en línea. En este sentido, la seguridad de la información debería verse como un habilitador más que como un inhibidor, tanto para las empresas como para las personas. Es en este contexto que la IA modular podría llegar a jugar un papel fundamental, gracias a su capacidad en el manejo de metadatos y el aprendizaje continuo. 
Por último, los Estados Unidos disfrutaron ventajas sustanciales en innovación y fuerza económica en la segunda mitad del siglo pasado. Muchas de las innovaciones en la IA ocurrieron en los Estados Unidos y los beneficios que asisten a estas innovaciones se acumularon primero en ese país. No obstante, estas innovaciones se están difundiendo con rapidez, especialmente con el fuerte impulso académico y comercial para democratizar la IA. El aumento de expertos en IA e innovadores en otras naciones (por ejemplo, China) es probablemente la señal más indicativa que apunta a la pérdida de la ventaja de los Estados Unidos como primer jugador en el terreno de la IA, lo cual tendría, en el mediano y largo plazo, efectos geopolíticos y geoeconómicos considerables.

\section{Referencias}

Allen, G., \& Chan, T. (2017). Artificial Intelligence and National Security. Belfer Center for Science and International Affairs.

Álvarez, C. (2017). Geopolítica vertical y el fenómeno de urbanización de la guerra en el Siglo XXI. Ensayos en Defensa y Seguridad, 11, 8-38.

Álvarez, C., Santafé, J. \& Urbano, O. (2017). Metamorphosis Bellum: ¿Mutando a guerras de quinta generación? En C. Álvarez (Ed.), Escenarios y Desafíos de la Seguridad Multidimensional en Colombia (pp. 145-248).

Álvarez, C., \& Zambrano, J. (2017). Globalización desviada: plataforma de convergencia criminal. En C. Álvarez (Ed.), Escenarios y Desafíos de la Seguridad Multidimensional en Colombia (pp. 249-307).

Ayoub, K., \& Payne, K. (2016). Strategy in the Age of Artificial Intelligence. Journal of Strategic Studies, 39(5-6), 793-819.

Brynjolfsson, E., \& McAfee, A. (2014). The Second Machine Age: Work, Progress, and Prosperity in a time of Brilliant Technologies. W.W. Norton.

Cipolla, C. (2003). Before the Industrial Revolution: European Society and Economy from 1000-1700. W.W. Norton. 
Cooper, C., \& Kaplinsky, R. (1989). Technology and Development in the Third Industrial Revolution. Routledge.

Haegele, M. (2019). World Robotics 2017 Service Robots. International Federation of Robotics.

Headrick, D. (2009). Technology: A World History. Oxford University Press.

Herrendorf, B., Rogerson, R., \& Valentinyi, A. (2014). Growth and Structural Transformation. En P. Aghion \& S. Durlauf (Eds.), Handbook of Economic Growth, 2, 855-941.

Jensen, G., \& Wiest, A. (2001). War in the Age of Technology: Myriad Faces of Modern Armed Conflict. New York University Press.

Jörg, B. (2016). A History of the Global Economy: From 1500 to the Present. Cambridge University Press.

Kelley, K. (2014, 27 de octubre). The Three Breakthroughs That Have Finally Unleashed AI on the World. Wired Magazine. https://www.wired.com/ 2014/10/future-of-artificial-intelligence.

Keynes, J. (1963). Essays in Persuasion. W.W. Norton.

Manyika, J., Lund, S., Chui, M., Bughin, J., Woetzel, J., Batra, P., Ko, P., \& Sanghvi, S. (2017). Jobs Lost, Jobs Gained: Workforce Transitions in a Time of Automation. McKinsey Global Institute.

Marx, K. (1973). Grundrisse: Foundations of the Critique of Political Economy. Vintage Books.

Ricardo, D. (2004). On the Principles of Political Economy and Taxation. Dover Publications.

Sattikar, A., \& Kulkarni, D. (2012). A Role of Artificial Intelligence Techniques in Security and Privacy Issues of Social Networking. International Journal of Computer Science Engineering \& Technology, 2(1), 792-795.

Schwab, K. (2016). The Fourth Industrial Revolution. World Economic Forum.

Søgaard, M., Jørgensen, U. \& Clausen, C. (2009). The Social Shaping Approach to Technology Foresight. Futures, 41, 80-86.

Singer, P. (2004). Wired for War: The Robotics Revolution and Conflict in the 21st Century. Penguin.

Skilton, M., \& Hovsepian, F. (2017). The 4th Industrial Revolution: Responding to the Impact of Artificial Intelligence on Business. Palgrave Macmillan. 
Teng, J. (2014). Musket, Map and Money: How Military Technology Shaped Geopolitics and Economics. Versita.

Wittmann, M. (2019). World Robotics 2019 Industrial Robots, Frankfurt: International Federation of Robotics.

Zafirovski, M. (2011). The Enlightenment and Its Efects on Modern Society. Springer. 